\title{
Digitizing Conventional Learning Materials: Production Phase
}

\author{
Stella Stefany ${ }^{1}$, Rijanto Purbojo ${ }^{2}$ \\ Distance Learning of Communication Science, Universitas Pelita Harapan, Indonesia ${ }^{1}$ \\ Master of Instructional Technology, Universitas Pelita Harapan, Indonesia ${ }^{2}$ \\ \{ctella.stefany@uph.edu ${ }^{1}$,rijanto.purbojo@uph.edu²
}

\begin{abstract}
Digitalization of Education field has been highlighted as main initiative to improve the quality of human capital as part of national roadmap program "Making Indonesia 4.0" (interpreted as industry 4.0) which has been officially launched on April 2018. Education Ministry launched the national online learning system as initiative to provide wider access of education. As new method of learning, it requires an innovative strategy to transform the system. One of the most major things is to produce high-quality online course module as substitution of face-to-face (FtF) learning method. This research is aimed to identify and analyze the conversion steps of learning materials and compare the learning outcomes. Digital content in this study refers to online course module for higher education. FGD was conducted with 10 participants to evaluate design and development process of digitizing conventional materials. During January and April 2019, 97 students were involved in this research, they are equally assigned to two types of learning modes; fully online and onsite classroom in which taught by the same lecturer with same learning objectives, assessment materials, and assignments. The finding is there were no significant differences among the student learning outcomes of on-site and distance learning modes. We concluded that the strategy and process of converting regular course module into the digital course one has no effect on the learning outcomes in both blended learning and distance learning strategy.
\end{abstract}

Keywords: digitalization; online learning; digital communication; digital content; online course module; instructional design; media richness

\section{Introduction}

Making Indonesia 4.0 launched by President Joko Widodo by April 2018. The significant change in the term of 4.0 is Internet of Things, cyber physical system, and network[1]. Entering the industrial revolution 4.0, education field mentioned as one of the main stakeholders to support this industrial revolution. It stimulated the launching of national online learning system (SPADA). By the end of 2018, permit to operate the new online study programs given to 7 universities in Indonesia. As a new learning method, it requires changes in teaching and learning culture. The emerging of new ICT and education technologies insisted higher educational to expand learning opportunities for diverse students or learners in 
online education to cross the geographical and time-distance barriers[2]. Theory of online learning interaction, suggests that the various forms of student interaction can be substituted for each other, depending on cost, content, learning objectives, convenience, technology and available time. However, the substitutions do not result in decreases in the quality of the learning results. Anderson[4] also mention that sufficient levels of deep and meaningful learning could be obtained, only in the condition that, at least, one among three types of learning interaction (student-teacher; student-student; student-content) has occurred at very high levels. Whereas, the other two may be offered at minimal levels or even eliminated without degrading the educational experience.

Online learning requires students' self-regulated learning while using the LMS that needs careful consideration while providing online learning to the students. The key issue is how to transform the FtF class learning experience into online learning environment delivered by an LMS. There are at least two perspectives to answer this issue: the perspective of media utilization in computer-mediated communication (CMC) and Instructional Systems Development (ISD). Further, media utilization requires digital content development as well. This study tries to construct the model of digitizing conventional materials into digital ones and its implication to the learning outcomes. An elaboration on how the process of digitizing material for students in higher education was done and the teaching delivery is implemented are also presented in this paper. This study will emphasize more in the student - content type of interaction.

\section{Method}

This study aims to answer: (1) Design and development process of digital learning materials evaluation; and (2) Comparison the effectiveness of digital learning material to online classes and onsite class, as shown on figure 1 below.

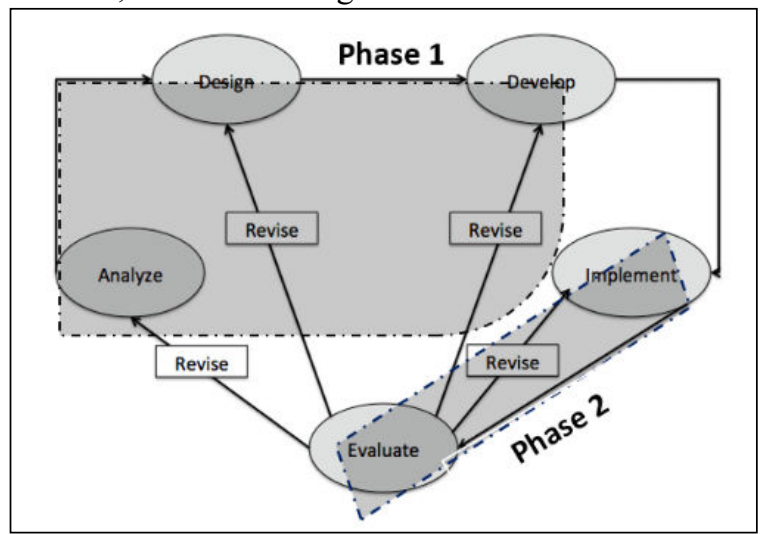

Fig 1. Research Method

The finding will clarify the relation between designing and developing online course module and the effectiveness of the digital media to support onsite and online distance learning course. By considering the situatedness of a realistic learning environment, it is decided to adopt program evaluation research methodology, defined as a research done by a teacher which designed to make decision about the program[5]. Thus, this study is not merely 
prototyping the digital course module, but also to test the result of design of digital course module into the actual teaching and learning environment.

Phase 1. Evaluate design and development process of digitizing learning materials, Focus group discussion (FGD) sessions conducted to 10 participants, who actively work in online learning industries as a team. It consists of subject matter experts (SME), instructional designers, education technology, learning management specialist, audio-visual integrator (AVI), lecturer and content specialists from several universities that have a license to operate online study program. The discussion points are to find: (1) the composition of the professional team member and their roles and responsibility, (2) the most appropriate Syllabus Design, (3) the components of an online course module, (4) the best process of creating course module, (5) the criteria to standardize the video lectures.

Phase 2. Quantitative Comparative Study (T-Test), Statistical analysis was done to compare students' activities and outcome between two types of learning modes: onsite and online classroom. The researcher delivered one course with two types of learning modes to 97 students between January to April 2019. We used 2 online classes in the distance learning program and 1 onsite classroom in the FtF class. These 2 classes are supported by Moodle LMS. Student's activities in the LMS is recorded and monitored daily. Their learning outcomes are assessed by parallel objectives and essay test, and some individual assignments. These assessment activities are graded by using the same answer key and grading rubrics. The total grades are weighted and later used to determine their completion of study in this course.

\section{Result and Discussion}

\subsection{Design and development process of digitizing learning materials evaluation (FGD)}

\section{a) Role and Responsibility of the Professional Team Member}

By using an LMS, an on-site classroom can be substituted into an online one. However, there is an ongoing debate about whether it is the use of a particular delivery technology or the design of the instruction that improves learning[6]. Hence, significant parties involved in online learning need to be changed. Lecturers need to be competence to create activities, lecturing, assessment to achieve students' competences in every course[7]. In fact, many teacher remain resistant to the intrusion of technology in classroom[8]. From the FGD session, it is identified that there are several professional members and its specific roles and responsibilities (see Table I below). To enforce that digitizing the learning process, leadership needs to consider centralizing these roles and responsibilities in one working unit. A center for online learning at least consists of SMEs, Instructional Designers, Audio Visual Integrators, LMS administrators, Education Technology Specialists, and General Administration staff members.

\section{b) Syllabus Design}

According to Corbin[9], to promote higher-order thinking on the Website, online learning must create challenging activities that enable learners to link new information to the old ones, acquire meaningful knowledge, and use their metacognitive abilities; hence, it is the instructional strategy and not only the technology that determines the quality of learning. Qin[10], in her previous study mentioned that students suggested that the delivery medium is 
not the only determining factor in the quality of learning; but rather, the design of the course determines the effectiveness of learning [11]. To start the design of an online course, syllabus determined as the first thing to be constructed. Syllabus design consists of 3 steps: defining the competency standard and mapping, creating concept mapping, and creating program mapping. Dick[13] explained that range from a simple online syllabus, at least consists of content, instruction, interaction and assessment. Another steps of designing a syllabus which is also associated with learning task analysis, called sequential concept mapping. There are several options of concept mapping models: (1) flow chart, (2) hierarchies, (3) circle or spider webs with line connecting the concept to show each relationship[14].

\section{c) Components of Online Course Module}

Media richness and Social Presence Theories should be considered in deciding which media to use in digitizing materials. Media richness refers to the capacity to convey a broad spectrum of information, including immediacy of feedback, multiple cues, language variety and personal focus[15]. It argues that there is a match between the equivocality of a message situation and the richness of medium which to address that related to communication effectiveness [16]. In most online education courses, a combination of media and technology are used. There is no single technology can address all the teaching and learning requirements that will satisfy the need of learners. The more media alternatives are provided, the more effective online education course is likely to be for a wider range of students[17]. Social Information Processing Theory of Computer-Mediated Communication (CMC) has become widely used framework to explain and predict differences between text-based and offline communication As written in Channel Expansion Theory[19], individuals gain more experiences with a particular medium, at last, the medium itself becomes richer for them. These various communication media differed in their capacity to transmit classes of nonverbal communication as addition to verbal ones. In this study, we concluded that the components of online course module at least should consist of (1) presentation max 15 slides/session, (2) reading materials, (3) Forum Discussion topics for each session, (4) Lecturing videos max 20 minutes, (5) question bank, (6) assignment, (7) assessment, (8) rubrics and answer keys, (9) digital references, (10) animation/gamification.

\section{d) Process of Creating Online Course Module}

According to Social Presence Theory, media can also be distinguished by the extent to which they permit communicators to experience others as being physically or psychologically present. As for online learning, physically present as FtF lecturing should be mediated by both video lectures (asynchronous) or video conferencing (synchronous). The finding is that video lessons are vital to the success of an online course [21]. The study about students' insights on the use of video lectures in an online class found that students' satisfaction with the course videos had a strong relationship with a positive overall learning experience [22]. Although good course videos alone cannot guarantee the success of a course, for sure, the use of videos with bad design and poor quality more likely get poor ratings from students on the whole course. By referring to the media richness theory, it is then understandable that in online learning that utilize internet is considered as "lean" media in which may reduce the successfulness of task completion, compared to the onsite or FtF classroom. So, it is very important to define the process of creating online course module. The process started by the time SME design the syllabus set and discuss with Instructional Designer to construct the 
learning scenario. Then, a SME will provide all materials as required in the online course module components. While a SME and AVI team working collaboratively in recoding each video session, general administrator will assist the SME in digital document templating both reading and presentation files (supporting materials) in each learning session. After recording, AVI team will do editing to produce high-quality video. Both final output of video lectures and supporting materials need to be deployed by SME in LMS. The LMS administrator deploy and activate the online course module in LMS as soon as the SME confirmed the quality of the online course module. The LMS administrator also enroll the students in the course. By the end of the semester the LMS administrator makes backup files of the online course module regularly.

\section{e) Standardize Criteria of Video Lectures}

To produce a good course design that utilizing media, Association of Educational Communication Technology introduce the terminology of Instructional System Design (ISD), defined as an organized procedure that is includes the steps of analyzing, designing, developing, implementing and evaluating instruction [23]. Instructional Development (ID) consists of at least five major activities: (1) analyze setting and audiences (learners), (2) design the specific efficient, effective and relevant learning environment, (3) development learning materials, (4) implementing result instruction, and (5) evaluation of the result development. These activities referred as ADDIE and labelled as basic ID Model [24]. As a finding on their previous study, Ou, Joyner \& David [21] emphasized that video lessons are vital to the success of an online course. The study about students' insights on the use of video lectures in an online class found that students' satisfaction with the course videos had a strong relationship with a positive overall learning experience[22]. The use of video in digital content is assumed to increase the social presence in the online learning module. Result of this study showed that there are 4 types of video lecturing: (1) Trailer, as an introduction with maximum duration 4-6 minutes, (2) lecturing as voice-over or talking-head presentation max. 20 minutes, (3) Learn from expert as talkshow with max. 20 minutes and (4) skill based video, as cyber lab, screen casting, simulation, max. 20 minutes.

\subsection{Comparison of students' activities and outcome between online and onsite class}

The second section of the data collection results presented on table IV below. These results are taken from the second phase of data collection.

Table 1. Descriptive Statistic Learning Outcomes

\begin{tabular}{cccccc}
\hline Learning Delivery Modes & N & Min. & Max. & Mean & Std. Deviation \\
\hline Distance 1 & 28 & 12.22 & 95.50 & 67.05 & 24.81 \\
Distance 2 & 26 & 10.40 & 96.68 & 65.82 & 21.22 \\
Onsite & 43 & 22.35 & 85.47 & 65.50 & 12.30 \\
TOTAL & 97 & 10.40 & 96.68 & 66.04 & 18.90 \\
\hline
\end{tabular}

Depicted by table IV above, the mean score of all participants was $66.04(N=97$, $S . D .=18.89)$. This mean score is not normally distributed, with skewness of $-1.032(S E=0.245)$ and kurtosis of $1.185(S E=0.485)$. The descriptive statistic shows that the mean score from the total data is above 60 (max. 100 points) which is the passing grade of the course study. Further, we observed that the mean score of two online distance learning classes and one onsite classroom are also equal. There is a slightly lower score of the standard deviation of the 
onsite classroom learning outcomes compared to two online distance learning classes, which indicates a wide variation of the data points. It means that the variety of learning outcomes in onsite classroom is less than in the online distance learning class. Moreover, we could also see that the maximum score of onsite classroom is slightly lower than the other two online distance learning classes. This could be related with the issue of self-regulated learning in online learning where the student finds more difficulty to maintain their motivation to selfstudy the material, than in the onsite classroom setting where there is a direct guidance from the teacher. Thus, online distance learning possibly elicits a learning situation where the individual differences of the students are exercised during the learning process, hence more variation on the learning outcomes. The Mann-Whitney Test is conducted to compare two online classes: Distance $1(N=28$, Mean $=67.05, S . D .=24.81)$ and Distance $2(N=26$, Mean $=65.82, S . D .=21.22)$ and indicated that there is no significant difference of the average mean rank of learning outcomes from both online distance classes $(U=321, \mathrm{z}=-.744, p=.46)$. By this finding, we concluded that that both online distance classes have equal learning outcomes, although the mean score indicated above averages scores but they are still in the mediocre level. This finding showed that although the participants of both classes are different, the average learning outcomes of two online distance learning classes are equal. Further, The Kruskal-Wallis Test is done to provide comparison study among 2 online distance learning classes and 1 onsite classroom $(N=43$, Mean $=65.50, S . D .=12.30)$. It is found that there is no significant difference between mean ranks of the learning outcomes from those three classes $(H(2)=1.855, p=.365)$. This is the key finding for this study, because it violates our original prediction where the learning outcomes of onsite classroom would exceed the online distance learning classes. This is a confirmation that the 2 online distance learning classes have equal learning outcomes to the onsite classroom, which is in average still above 60 points. From the perspective of instructional systems development (ISD), these findings support our online course design expectation. It is not only confirming that it is possible to obtain above average learning outcomes in online distance learning process, but also a thorough Instructional design that embedded in ISD yielded a meaningful course design. Linking to the result of FGD, these findings confirm the value of a meaningful online course distance learning which is comparable to onsite classroom learning mode. Taking the media richness and social presence theories into the consideration, the onsite classroom learning has failed to maximize the value of online course module and its video as educational media. Further analysis must be done to confirm this new prediction. It is possible that the students in onsite classroom is confused, feeling a mismatch between the equivocality of a message situation in the classroom and the richness of the digital content in the online course module as mentioned earlier by Mcquail[16]. It is suggested to design less social presence in an online course module for blended learning strategy which still emphasize classroom direct instruction by having a limited number or shorter duration of video lecturer files.

\section{Conclusion}

Above findings conclude that a well-design online course module adds values to online and onsite classes. The effectiveness of the digital content in an online course module could be obtained not only by considering the media design perspective, media richness and social presence theory but also the instructional design process. The instructional design model is intended to create a meaningful learning experience, which will provide a rationale in determining how rich the media. The social presences in online learning module could be 
obtained by producing and embedding short duration but rich content video files, for example 5-10 minutes, in the online course. In the case of online distance learning, social presence could be added by utilizing planful video conferencing sessions.

Further studies need to be conducted to objectively measure the entry level of students in both onsite and online distance learning classes. It is very important to know the characteristic of the student and improvise the learning activities according to the need of the student personally. Secondly, there is a strong need to closely monitor the daily student activity in the online distance learning class, by utilizing the learning analytic features of the LMS. Improvement of student's digital literacy skills during learning process through online learning module and its digital content such as video lectures has not yet studied in this research. Lastly, it is suggested to highlight social interaction of distance learning students to study their peers and instructors virtual relationship.

\section{Acknowledgment}

Highest gratitude to Ms. Stephanie Riady who trusted us opportunity to develop online learning in UPH and to Prof. Paulina Pannen, M. Ls and Dr. Uwes A. Chaeruman, M. Pd., from education ministry of Indonesia for all guidance and national-wise-direction our university. Everyone who has provided us extensive personal and professional guidance and contributed a great deal of data needed. Particularly for Esther Ida Krisanti and Anita Suhendro for such a great supportive and collaboration in producing significant improvement of our online learning.

\section{REFERENCES}

[1] A. Rojko, "Industry 4.0 concept: Background and overview," Int. J. Interact. Mob. Technol., vol. 11, no. 5, pp. 77-90, 2017.

[2] F. Patel, Online Learning: An Educational Development Perspective. New York, NY: Distance education--Development, 2014.

[3] A. Nurcan and T. Tuğba, "The Impact of Motivation and Personality on Academic Performance in Online and Blended Learning Environments," Educ. Technol. Soc., vol. 21, no. 3, pp. 35-47, 2018.

[4] T. Anderson, The theory and practice of online learning, 2nd Ed. Edmonton: AB: AU Press, 2011.

[5] J. W. Santrock, Educational psychology, 5th ed. New York: McGraw-Hill, 2011.

[6] R. E. Clark and R. E. Clark, "Challenges for Institutional Theory - Management," vol. 53, no. 4, pp. 445-459, 2009.

[7] R. Purbojo, "Role of the University lecturer in an analysis of Moodle features utilized in a blended learning," in Educational Technology to Improve Quality and Access on a Global Scale, 2018, pp. 227-244.

[8] D. Buckingham, "Media education goes digital: An introduction," Learn. Media Technol., vol. 32, no. 2, pp. 111-119, 2009.

[9] H. J. Corbin, "The learning camera: A personalized learning model for online pedagogy in human services education.,"J. Technol. Hum. Serv., vol. 37, no. 4, pp. 334-346, 2019.

[10] Q. Sun and G. Ganesh, "Developing and Teaching an Online MBA Marketing Research Class: Implications for Online Learning Effectiveness," J. Educ. Bus., vol. 89, pp. 337-345, 2014.

[11] A. Rovai, "Building Sense of Community at a Distance," vol. 3, no. 1, pp. 1-16, 2002.

[12] S. Bulfin and K. McGraw, "Digital literacy in theory, policy and practice: old concerns, new opportunities," 2015, pp. 266-281.

[13] W. Dick, L. Carey, and J. O. Carey, The systematic design of instruction, 8th ed. Boston: 
Pearson, 2015.

[14] J. D. Novak, Learning, creating, and using knowledge: concept maps as facilitative tools in schools and corporations, 2nd ed. New York, NY: Routledge, 2010.

[15] N. Park, J. E. Chung, and S. Lee, "Explaining the Use of Text-Based Communication Media: An Examination of Three Theories of Media Use," CyberPsychology, Behav. Soc. Netw., vol. 15, no. 7, pp. 357-363, 2012.

[16] D. McQuail, Communication theory \& research: an ECJ anthology. London; Thousand Oaks, Calif: SAGE, 2005.

[17] M. Moore and G. Kearsley, Distance Education: A System View, 3rd ed. Belmont: Cengage, 2011.

[18] B. Hersh, "The Paradox of Social Information Processing Theory," Int. J. Sci. Soc., vol. 3, no. 1, 2012.

[19] T.-S. Hew, S. A. Kadir, and S. Latifah, "Applying Channel Expansion and Self-Determination Theory in predicting use behaviour of cloud-based VLE.," Behav. Inf. Technol., vol. 36, no. 9, pp. 875-896, 2017.

[20] M. A. Carter, "A study of students' perceptions of the online component of a hybrid postgraduate course," Soc. Behav. Sci., vol. 84, pp. 558-568, 2013.

[21] C. Ou, D. A. Joyner, and A. K. Goel, "Designing and developing video lessons for online learning: A seven-principle model," Online Learn. J., vol. 23, no. 2, pp. 82-104, 2019.

[22] N. I. Scagnoli, J. Choo, and J. Tian, "Students' insights on the use of video lectures in online classes,” Br. J. Educ. Technol., vol. 50, no. 1, pp. 399-414, 2019.

[23] B. Seels and R. Richey, Instructional Technology: The definitions and domains of the field. Washington, DC: Association for Educational Communications and Technology, 1994.

[24] K. L. Gustafson and R. M. Branch, Survey of Instructional Development Models. Fourth Edition. 2002. 